\title{
Anesthesia and enhanced recovery in subxiphoid video-assisted thoracoscopic surgery
}

\author{
Zhigang Chen ${ }^{1 \#}$, Jason M. Ali ${ }^{2 \#}$, Huan $\mathrm{Xu}^{1}$, Lei Jiang ${ }^{1}$, Giuseppe Aresu ${ }^{2}$ \\ ${ }^{1}$ Department of Thoracic Surgery and Anaesthesia, Shanghai Pulmonary Hospital, Tongji University, Shanghai 200433, China; ${ }^{2}$ Thoracic Surgery, \\ Royal Papworth Hospital NHS Foundation Trust, Papworth Everard, Cambridge, UK \\ Contributions: (I) Conception and design: All authors; (II) Administrative support: None; (III) Provision of study materials or patients: All authors; \\ (IV) Collection and assembly of data: All authors; (V) Data analysis and interpretation: All authors; (VI) Manuscript writing: All authors; (VII) Final \\ approval of manuscript: All authors. \\ "These authors contributed equally to the work. \\ Correspondence to: Lei Jiang, MD. Department of Thoracic Surgery, Shanghai Pulmonary Hospital, Tongji University, Shanghai 200433, China. \\ Email: jiangleiem@aliyun.com.
}

\begin{abstract}
Subxiphoid video-assisted thoracoscopic surgery (VATS) is a surgical approach in minimally invasive thoracic surgery that aims to aid enhanced recovery by reducing postoperative pain by avoiding instrumentation of the intercostal spaces. Access through a subxiphoid port presents challenges for both the surgeon and anaesthetist. Particularly for left sided procedures, the heart can be compressed resulting in arrhythmia and haemodynamic compromise. The anaesthetic team play an important role in ensuring the success of subxiphoid VATS procedures. The key is continuous and comprehensive monitoring for circulatory disturbance and arrhythmia intraoperatively. Should arrhythmia develop it is important that it is managed rapidly and effectively in such a manner to minimize haemodynamic disturbance. In this article, important considerations for anaesthesia in subxiphoid VATS procedures is presented and solutions presented.
\end{abstract}

Keywords: Anaesthesia; subxiphoid; video-assisted thoracoscopic surgery (VATS); subxiphoid VATS (SVATS); enhanced recovery

Submitted Nov 08, 2018. Accepted for publication Nov 16, 2018.

doi: $10.21037 /$ jtd.2018.11.90

View this article at: http://dx.doi.org/10.21037/jtd.2018.11.90

\section{Anaesthesia and enhanced recovery in subxiphoid video-assisted thoracoscopic surgery (VATS)}

Thoracic surgery is evolving. A large thoracotomy is no longer the standard surgical access to the pleural cavity. VATS has become the norm and with it, improved patient outcomes and faster return to normal activity (1-3). However, VATS surgery is still associated with significant pain, and a risk of developing chronic pain due to instrumentation of the intercostal space. In an attempt to further improve outcomes in terms of pain and recovery from thoracic surgery a more recent development has been the use of the subxiphoid approach. In this approach, a vertical incision is made in the subxiphoid space and the pleural cavity is entered at this level (Figure 1). This approach then avoids any instrumentation of the intercostal spaces avoiding the potential for nerve compression and injury (4). To date, there has been one randomised controlled trial comparing subxiphoid VATS (SVATS) to standard VATSin patients undergoing bullectomy and pleurectomy. They observed a significant reduction on postoperative pain in the SVATs group which was associated with earlier mobilisation and reduced risk of complication (5). The SVATS approach has become increasingly popular, and there are reports of a wide range of operations being performed from anatomical segmentectomies to pneumonectomies (6-15).

Thoracic surgery is usually performed in the context 


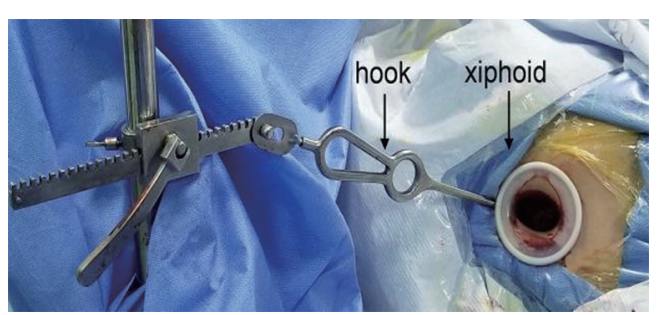

Figure 1 Subxiphoid access port for surgery with a patient in the right lateral decubitus position.

Table 1 Contraindications for subxiphoid VATS surgery

Absolute
Significant cardiomegaly
Severe arrhythmia
Impaired left ventricular function
Relative
Body mass index $>30 \mathrm{~kg} / \mathrm{m}^{2}$
Age $>80$ years
More complex and prolonged surgery

VATS, video-assisted thoracoscopic surgery.
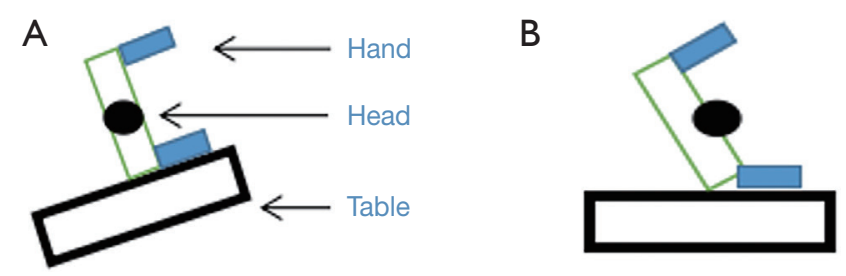

Figure 2 Optimal patient positioning with the patient tilted away from the lateral decubitus position which can be achieved by either tilting the operating table (A), or tilting the patient (B).

of well-developed enhanced recovery programs which further aids the benefit of the SVATS approach $(16,17)$. However, SVATS presents certain perioperative challenges for the thoracic anaesthetic team. Particularly in left sided procedures, the patient's heart may need to be displaced which can cause haemodynamic compromise and dysrhythmia.

\section{Preoperative preparation}

As for all surgical patients, pre-operative assessment is an important stage in preparation for surgery. Patients should be assessed by experienced thoracic anaesthetists with focus on documenting past medical history and performing physical examination. Basic laboratory investigations and pulmonary function tests are performed to ensure operability (18).

In the context of SVATS, the anaesthetic team should ensure additional attention is placed on assessing cardiovascular function. We would advocate that patients should have an electrocardiogram performed to assess for any dysrhythmia, and if there is any concern over cardiovascular function, echocardiography and exercise testing should be considered. In patients with compromised cardiovascular function, the operative approach should be reconsidered to minimize the risk to the patient (Table 1).

\section{Intraoperative anaesthesia}

\section{Anaestbesia}

The choice between total intravenous anaesthesia or combined anaesthesia should be determined by the preference and experience of the anaesthetic team. Anaesthesia should be induced, and patients intubated with a double-lumen endotracheal tube. The maintenance of sedation, analgesia and muscle relaxation during the surgery should the same as for standard VATS procedures.

\section{Patient positioning}

There are two commonly employed positions on the operating table for patients undergoing SVATS. To have adequate exposure of the subxiphoid region the patient needs to be tilted away from the lateral decubitus position. This can be achieved either by adjusting the angle of the operating table with the patient in the lateral decubitus position (Figure $2 A$ ) or positioning the patient tilted on the horizontal operating table (Figure $2 B$ ). It is important to pay attention during positioning of the patient to prevent injury to the brachial plexus and cervical spineby preventing excessive head extension and/or rotation by using appropriate support devices.

\section{Intraoperative monitoring}

Standard monitoring should be performed including electrocardiogram, oxygen saturations, end-tidal $\mathrm{CO}_{2}$ and central temperature. For SVATS, we would advocate insertion of an arterial line and, especially in case of 
left sided procedures, central venous line in order that arterial blood pressure and central venous pressure can be monitored during the procedure, and blood gas analysis can be performed. These additional measures are employed to allow monitoring of the potential haemodynamic instability that can occur (19).

\section{Intraoperative fluid management}

Patients should receive intravenous fluid throughout the procedure, however they should be administered conservatively. In SVATS, occasionally there may be greater traction and compression of the lung parenchyma due to the challenging angles sometimes required to perform the surgery, which can lead to higher risk of lung injury and postoperative pulmonary oedema. The fluid strategy employed should also consider the duration of pre-operative dehydration the patient has experienced and be titrated to intraoperative cardiovascular monitoring and operative blood losses.

\section{Ventilatory settings}

A protective ventilation strategy should be utilized-with the aim of minimizing tidal volume (tidal volume of $6 \mathrm{~mL} / \mathrm{kg}$ ) with a low positive pressure (positive end expiratory pressure $\left.5-10 \mathrm{cmH}_{2} \mathrm{O}\right)(20)$. The benefit of this strategy is not only to minimize the risk of lung injury, but also there is a reduced oscillation of the mediastinum during single-lung mechanical ventilation. This aids surgical access by providing a stable and optimal space for the procedure which reduces the pressure of the surgical instruments on the heart.

\section{Perioperative complications and their management}

\section{Arrbythmia}

Due to the hearts position in relation to the subxiphoid access, arrhythmia is a frequent occurrence in SVATSparticularly in left sided procedures. The various surgical instruments are designed with angles to minimize cardiac interference, but some compression of the heart is inevitable and can result in a range of arrhythmias. The majority of encountered arrhythmias recover spontaneously and rapidly following temporary suspension of the surgery. Due to the location of the heart, the arrhythmias experienced in left sided procedures tend to be more severe and may result in hypotension.

A range of arrhythmias are encountered. The most benign arrhythmias experienced are single atrial (Figure $3 A$ ) or ventricular (Figure 3B) ectopic beats. Occasionally, ventricular ectopic beats are followed by an escape rhythm and a more sustained arrhythmia (for example, Figure $3 C, D$ ). It is also possible to observe a mixture of atrial and ventricular ectopics (Figure 3E), and occasionally patients will go into atrial fibrillation which can lead to hypotension (Figure $3 F$ ). It has also been observed for patients to develop ventricular tachycardia (Figure $3 G$ ).

The aim intraoperatively is to prevent haemodynamic compromise and/or progression of any arrhythmia experienced-aiming to reduce the frequency of ectopy and treat haemodynamic compromise experienced as a result of tachy/brady arrhythmias. The majority of arrhythmias experienced are easily reversed by pausing the surgery. Occasionally, it may be necessary to administer anti-arrhythmic drug treatment, although this does not need to be continued following the surgery. It may also be necessary to cardiovert a patient out of a rhythm such as atrial fibrillation-although this can be performed at the end of surgery with the patient supine unless there is an urgent need to treat the arrhythmia. If the patient becomes hypotensive then more aggressive management may be required and the surgery should be paused to allow for recovery and/or treatment to be administered—as this will then reduce the compression on the heart that likely is precipitating the arrhythmia. If the patient remains hypotensive then fluid resuscitation may be required, guided by the central venous pressure and the next step would be to consider introduction of vasoactive drug therapy. At this point, it would be advisable to convert from SVATS to a standard approach in order to safely complete the surgery.

Continuous monitoring of arterial blood pressure and electrocardiography is essential during SVATS in order that the anaesthetic team can remain vigilant to diagnose and respond rapidly to any arrhythmia and/or haemodynamic compromise that develops during the course of the procedure.

\section{Hypoxemia}

Similar to the situation in standard VATS, desaturation is a common problem faced by patients on single lung ventilation. The commonest problem is malposition of the double lumen tube due to migration. Other problems include ventilation/perfusion mismatch due to single lung 
A

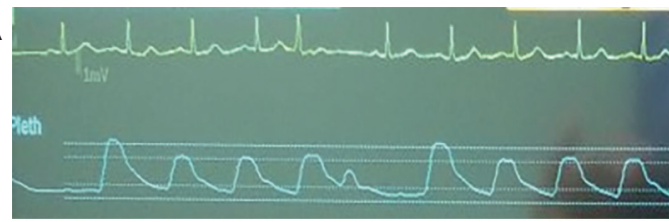

C

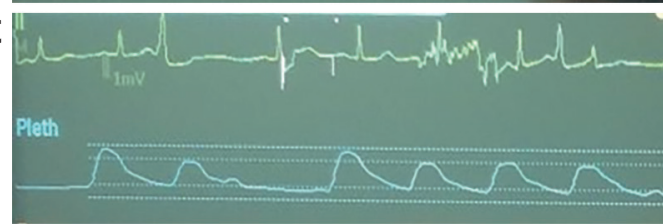

E
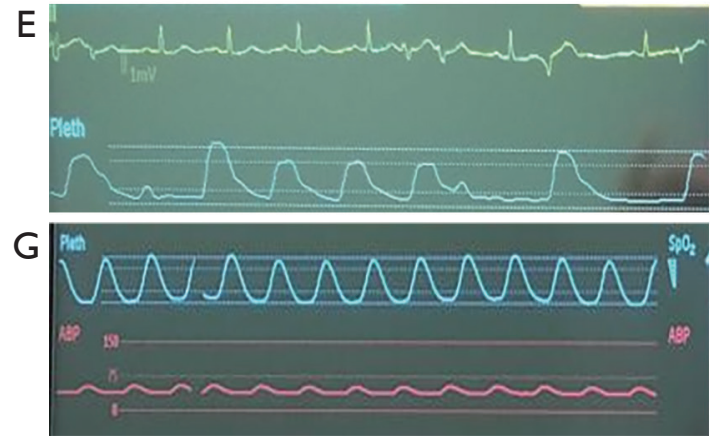
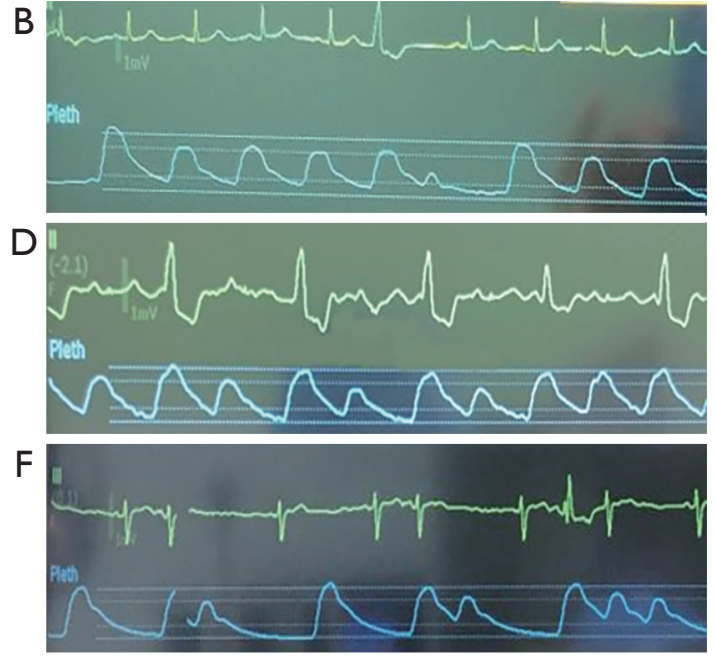

Figure 3 Example arrhythmias experienced during subxiphoid VATS procedures. (A) Atrial ectopy, (B) ventricular ectopy, (C,D) ventricular ectopy leading to escape rhythms, (E) mixture of atrial and ventricular ectopy, (F) atrial fibrillation and (G) ventricular tachycardia. VATS, video-assisted thoracoscopic surgery.

Table 2 Management of patients desaturating during subxiphoid VATS surgery

Gradual desaturation

Confirm the position of DLT is correct with fiber bronchoscopy

Recruitment maneuver of the ventilated lung

Increase $\mathrm{FiO}_{2}$ to 1.0

Increase blood pressure if it too low

Apply $5 \mathrm{cmH}_{2} \mathrm{O}$ PEEP

CPAP to the nonventilated lung

Acute desaturation

Two lung ventilation immediately and then identify the reason for hypoxemia

VATS, video-assisted thoracoscopic surgery; DLT, double-lumen tube; PEEP, positive end-expiratory pressure ventilation; CPAP, continuous positive airway pressure.

ventilation and the lateral decubitus position and underlying pulmonary disease in the ventilated lung. A series of steps is recommended when facing desaturation during SVATS procedures (Table 2).

\section{Postoperative extubation and analgesia}

At the end of the surgery, two-lung ventilation is restored, and an airway pressure of $35 \mathrm{cmH}_{2} \mathrm{O}$ is applied for 10 seconds as a lung recruitment manoeuvre. If muscle relaxant has been used and remains active, an anticholinesterase such as neostigmine can be administered as reversal. Once the patient regains consciousness and is able to maintain an airway, they can be extubated and transferred to the recovery room and eventually discharged to the ward.

Although the aim of SVATS is to minimize the postoperative pain incited, it is essential that pain management is optimized as after all surgery to observe the maximal benefit and facilitate enhanced recovery. A range of approaches is adopted but usually includes combination of intravenous analgesia and regional nerve blocks, for example thoracic epidural or more commonly, paravertebral infusions $(21,22)$. Adequate antiemetic should be prescribed in order to minimize the complications associated with opiate analgesia. For patients with a history of vomiting or vertigo, the preventive use of dexamethasone and reducing intraoperative related drugs can be advantageous in preventing vomiting and nausea. 
Table 3 Example postoperative analgesia protocol to facilitate enhanced recovery following subxiphoid VATS procedures

\begin{tabular}{|c|}
\hline Day 0 in recovery \\
\hline Flurbiprofen axetil $50 \mathrm{mg}$ IV after arrival in recovery \\
\hline $\begin{array}{l}\text { Start IV PCA: sufentanil } 50-100 \mu \mathrm{g}+\text { flurbiprofen axetil } 100 \mathrm{mg} \\
\text { + tropisetron }\end{array}$ \\
\hline $\begin{array}{l}\text { If patients remain in pain, give bolus of IV sufentanil 5-10 } \mu \mathrm{g} \\
\text { but observe for respiratory depression }\end{array}$ \\
\hline $\begin{array}{l}\text { Reduce or avoid flurbiprofen axetil in patients who have } \\
\text { hypertension }\end{array}$ \\
\hline Day 0 HDU \\
\hline Continue PCA \\
\hline IV metoclopramide $5-10 \mathrm{mg}$ if nausea and vomiting \\
\hline $\begin{array}{l}\text { If severe nausea or vomiting then convert to transdermal } \\
\text { fentanyl patch }\end{array}$ \\
\hline Day 1 ward \\
\hline Continue PCA \\
\hline IV metoclopramide $5-10 \mathrm{mg}$ if nausea and vomiting \\
\hline Day 2 ward \\
\hline $\begin{array}{l}\text { Step down to oral analgesia if tolerated: oxycodone } \\
\text { hydrochloride } 5 \mathrm{mg} \text { and acetaminophen } 325 \mathrm{mg} \text { (Tylox) QDS }\end{array}$ \\
\hline Day 3 ward \\
\hline Continue Tylox QDS \\
\hline Patient remains on this post-discharge \\
\hline $\begin{array}{l}\text { If ongoing pain, reviewed by anaesthetic team for escalation of } \\
\text { therapy }\end{array}$ \\
\hline
\end{tabular}

VATS, video-assisted thoracoscopic surgery; PCA, patient controlled analgesia; HDU, high dependency unit; QDS, quaque die.

An example post-operative analgesia protocol that is utilized in Shanghai Pulmonary Hospital, China, is presented in Table 3. It has been designed to reduce the rate of complications and accelerate mobilization of the patient following surgery.

\section{Summary}

Subxiphoid VATS is a recent development in thoracic surgery that aims to aid enhanced recovery by reducing postoperative pain. The anaesthetic team play an important role in ensuring the success of these surgeries. The key is continuous and comprehensive monitoring for circulatory disturbance intraoperatively. Should arrhythmia develop it is important that it is managed rapidly and effectively in such a manner to minimize haemodynamic disturbance.

\section{Acknowledgements}

None.

\section{Footnote}

Conflicts of Interest: The authors have no conflicts of interest to declare.

\section{References}

1. Demmy TL, Nwogu C. Is video-assisted thoracic surgery lobectomy better? Quality of life considerations. Ann Thorac Surg 2008;85:S719-28.

2. Demmy TL, Curtis JJ. Minimally invasive lobectomy directed toward frail and high-risk patients: a case-control study. Ann Thorac Surg 1999;68:194-200.

3. Sugiura H, Morikawa T, Kaji M, et al. Long-term benefits for the quality of life after video-assisted thoracoscopic lobectomy in patients with lung cancer. Surg Laparosc Endosc Percutan Tech 1999;9:403-8.

4. Wang BY, Chang YC, Chang YC, et al. Thoracoscopic surgery via a single-incision subxiphoid approach is associated with less postoperative pain than single-incision transthoracic or three-incision transthoracic approaches for spontaneous pneumothorax. J Thorac Dis 2016;8:S272-8.

5. Li L, Tian H, Yue W, et al. Subxiphoid vs intercostal single-incision video-assisted thoracoscopic surgery for spontaneous pneumothorax: A randomised controlled trial. Int J Surg 2016;30:99-103.

6. Hatooka S, Shigematsu Y, Nakanishi M, et al. Subxiphoid approach for extracting a giant solitary fibrous tumour of the pleura. Interact Cardiovasc Thorac Surg 2017;25:834-5.

7. Fok M, Karunanantham J, Ali JM, et al. Subxiphoid approach for spontaneous bilateral pneumothorax: a case report. J Vis Surg 2017;3:146.

8. Karunanantham J, Fok M, Ali JM, et al. Subxiphoid single incision thoracoscopic surgery approach for thymectomy: a case report. J Vis Surg 2017;3:147.

9. Aresu G, Wu L, Lin L, et al. The Shanghai Pulmonary Hospital subxiphoid approach for lobectomies. J Vis Surg 2016;2:135-5.

10. Weaver H, Ali JM, Jiang L, et al. Uniportal subxiphoid video-assisted thoracoscopic approach for thymectomy: a 
case series. J Vis Surg 2017;3:169.

11. Fok M, Karunanantham J, Ali JM, et al. Subxiphoid approach for spontaneous bilateral pneumothorax: a case report. J Vis Surg 2017;3:146-6.

12. Ali JM, Kaul P, Jiang L, et al. Subxiphoid pneumonectomy: the new frontier? J Thorac Dis 2018;10:4464-71.

13. Karunanantham J, Fok M, Ali JM, et al. Subxiphoid single incision thoracoscopic surgery approach for thymectomy: a case report. J Vis Surg 2017;3:147.

14. Weaver H, Ali JM, Jiang L, et al. Uniportal subxiphoid video-assisted thoracoscopic approach for thymectomy: a case series. J Vis Surg 2017;3:169.

15. Ali J, Haiyang F, Aresu G, et al. Uniportal Subxiphoid Video-Assisted Thoracoscopic Anatomical Segmentectomy: Technique and Results. Ann Thorac Surg 2018;106:1519-24.

16. Giménez-Milà M, Martinez G, George S. Enhanced recovery programmes in thoracic surgery: how does the

Cite this article as: Chen Z, Ali JM, Xu H, Jiang L, Aresu G. Anesthesia and enhanced recovery in subxiphoid video-assisted thoracoscopic surgery. J Thorac Dis 2018;10(12):6987-6992. doi: $10.21037 /$ jtd.2018.11.90 future look? J R Soc Med 2018. [Epub ahead of print].

17. Scarci M, Solli P, Bedetti B. Enhanced recovery pathway for thoracic surgery in the UK. J Thorac Dis 2016;8:S78-83.

18. Kempainen RR, Benditt JO. Evaluation and management of patients with pulmonary disease before thoracic and cardiovascular surgery. Semin Thorac Cardiovasc Surg 2001;13:105-15.

19. Fischer $\mathrm{GW}$, Cohen E. An update on anesthesia for thoracoscopic surgery. Curr Opin Anaesthesiol 2010;23:7-11.

20. Brassard CL, Lohser J, Donati F, et al. Step-by-step clinical management of one-lung ventilation: continuing professional development. Can J Anaesth 2014;61:1103-21.

21. Gottschalk A, Cohen SP, Yang S, et al. Preventing and treating pain after thoracic surgery. Anesthesiology 2006; 104:594-600.

22. Joshi GP, Bonnet F, Shah R, et al. A systematic review of randomized trials evaluating regional techniques for postthoracotomy analgesia. Anesth Analg 2008;107:1026-40. 